East African Medical Journal Vol. 80 No. 7 July $200 \%$

A RANDOMISED CONTROLLED TRIAL OF EARLY INITIATION OF ORAL SEEDING AFTER CAESAREAN DELIVERY IN MULAGO HOSPITAL

D. Adupa, Obstetrician and Gynaecologist, Mulago Hospital, P.O. Box 7051 Kampala, Uganda. J. Wandabwa, Senior Lecturer and P. Kiondo Lecturer. Makerere Medical School, P.O. Box 7072 Kampala, Uganda

Request for reprinis to: Dr. J. Wandabwa. P.O. Box 29524. Kampala. Uganda

\title{
A RANDOMISED CONTROLIED TRIAL OF EARLY INITIATION OF ORAL FEEDING AFTER CAESAREAN DELIVERY IN MULAGO HOSPITAL
}

\section{ADUPA. J. WANDABWA and P. KIONDO}

\begin{abstract}
Background: The concept of early initiation of oral feeding after caesarean delivery is well tolerated by patients, yet not routinely practiced in Mulago Hospital. An effective postoperative dietary management schedule could have major implications on the management of maternal post-caesarean section mothers.

Objectives: To compare the effect of time initiation of oral feeding on acceptability, benefits and gastrointestinal functions in women who had undergone caesarean section in Mulago Hospital.

Design: A randomised controlled study.

Setting: Mulago Hospital.

Subjects: One hundred and ninety two women admitted on the postnatal ward after emergency or elective caesarean section for various indications and who satisfied the eligibility criteria were recruited and randomized into the study. Assignment to the early feeding group or routine feeding group was done randomly using a computer generated numbers. The early feeding group were encouraged to take sips of water within six to eight hours followed by oral soup or milk at least 150 millilitres at a time within 8 to 12 hours post operative under supervision. The routine group were managed by restricting oral intake for twenty four hours and administration of orals sips of water 24 to 48 hours post operative. The outcome measures were rate of ileus symptoms, post operative presence of bowel sounds, maternal pyrexia and acceptability and benefits of early feeding.

Results: The mean age, parity and gravidity were similar in the two groups. The study shows that women of the early feeding group had more rapid return of their bowel function with significant more shorter mean post operative time intervals to bowels sounds (24.2 hours versus 34.2 hours), passage of flatus (51.6 hours versus 62.1 hours) and bowel movement $(67.8$ hours versus 75.8 hours). The women who fed early, made more rapid recovery and expressed their interest in earlier hospital discharge. The findings significantly indicated that women in the early feeding group got out of bed (patient mobilisation) earlier $(p=0.001)$ than their control group ( 15.1 hours versus 17.8 hours). This could probably have been because of the adequate rehydration and improved early energy intake. In comparison those who were fed early required less number of bottles of intravenous fluids ( 5.0 bottles versus 7.0 bottles). The average hospital stay was similar and not statistically significant in both groups (5.5 days versus 6.0 days).

Conclusion: Early initiation of oral feeding after caesarean delivery is safe and well tolerated and can be implemented without an increase in gastrointestinal symptoms or paralytic ileus.
\end{abstract}

\section{INTRODUCTION}

Although the postoperative care of the obstetricgynaecologic patient may vary with the type of operation performed, there are certain traditions that most surgeons follow during the postoperative period. One long-standing caveat of post surgical care that relates to feeding of the abdominal surgery patient requires the patient to have normal bowel sounds and pass flatus before starting oral feeding. This practice is based on the assumptions that early feeding increases the incidence of nausea, vomiting and abdominal distension if bowel sounds have not returned before the initiation of oral feeding (1).

The current trend towards early discharge from hospital has led to the growing interest in patient management that allows more rapid recovery and resumption of normal activities. Therefore, concern has centred on the possible effects of early initiation of oral feeding on gastrointestinal functions, and thereby on patient hospital course and discharge. Evidence exists to support the clinical benefits of early postoperative oral feeding without significant increase in gastrointestinal complications (2). Therefore, time had 
come to put to test the notion of "Sleeping bowel" in the postoperative caesarean séction patient.

The concept of postoperative feeding: There is wide spread belief that postoperative ileus follows all intraabdominal surgical procedures as a result of anaesthesia and bowel manipulations. Its severity is thought to be a reflection of the amount of bowel manipulation, the amount of adhesions, duration of the surgery and whether the bowels are packed during surgery $(3,4)$.

Traditional (or routine) postoperative care maintains patients who have had uncomplicated abdominal surgery initially, is given "nothing by mouth" for 24 hours, then advanced to sips of clear liquid on the first postoperative day if bowel sounds are present and no abdominal distension. After the flatus and or stool is passed, the diet is accelerated as tolerated to a regular diet(4). This practice of withholding oral feeds was not scientifically tested until recently and was not evidence based. In fact, there is some evidence in the basic science and general surgery literature that disputes this surgical dogma and supports early oral feeding after surgery (1). Recent literature has shown that early oral feeding is associated with reduced protein store depletion, improved wound healing and faster recovery, with earlier hospital discharge and reduced costs (1).

In most cases the indications for caesarean section relate to an obstetric rather than a-medical problem. Also the majority of women who undergo caesarean delivery are relatively young, healthy, well nourished individuals who tolerate surgery well. The nature of caesarean section surgery, which includes minimal bowel manipulation, a low rate of pre-operative peritonitis and a relatively short duration of surgery allow a more rapid postoperative return of bowel function and quite more rapid patient recovery compared with other major abdominal surgeries or the $\mathrm{ill} / \mathrm{moribund}$ patients.

Early postoperative oralfeeding: The growing interest in early postoperative oral feeding may have been stimulated by the early resumption of oral diet intake recommended routinely after laparoscopic surgery $(3,6)$. It has been shown by recent studies that, immediate initiation of oral intake may not be unique to laparoscopy, but also may be well tolerated and even beneficial to patients who have undergone laparotomy and caesarean section (7-9). Furthermore, clinical observations (1,10,11), have suggested that the actual return of bowel function precedes clinically detectable signs of function by at least 24 hours, with the return of small-bowel function as, early as six hours after surgery in many cases. Therefore, the belief that postoperative ileus is a complete paralysis of the bowels with a complete absence of function is misleading. Regarding postoperative return of bowel function, array of factors may contribute to recovery among caesarean patients, including normal serum electrolyte levels, a low rate of pre-operative peritonitis, minimal bowel manipulation and a relatively short duration of surgery (12).
Early initiation of oral feeding has been advocated 'for a numbet of benefits. Its' nutritional value improved energy and protein intake in maintaining a positive caloric and nitrogen balance. This has the potential for improving the postoperative course by decreasing infection rates, improving wound healing, and preventing weight loss by maintaining nitrogen balance with administration of adequate calories (13).

The economic impact of early discharge from the hospital after an uncomplicated caèsarean section cannot be over looked. Early initiation of oral feeding after caesarean delivery is associated with a shorter hospital stay (2). The economic advantages of this regimen versus the continuing use of intravenous fluids are apparent. The cost of oral feeding is much less than the daily cost of 3 litres of intravenous fluids, giving sets, cannulas, nurses care and observations prescribed postoperatively, with the diet having many nutritional advantages over the standard I.V. fluids.

The purpose of this study was to compare the effect of time of initiation of oral feeding on acceptability, benefits and gastrointestinal functions in women who had undergone caesarean section in Mulago hospital.

Study design and Setting : This was a randomized controlled trial conducted in one of the postnatal wards of the department of obstetrics and gynaecology, Mulago Hospital.

\section{MATERIALS AND MÉTHODS}

Sample size determination: The primary outcome parameter was the rate of ileus symptoms. Twenty seven percent of patients who delivered by caesarean section developed mild ileus symptoms on the routine feeding schedule (2). To detect a $20 \%$ difference between the rates of $27 \%\left(\mathrm{P}_{2}\right)$ and assumed $47 \%\left(\mathrm{P}_{1}\right)$ of ileus symptoms, with an error of 0.05 and a power of $80 \%$, the Pitchard's formula for comparative studies was used to caiculate a total of 176 patients required. However, a target sample of (n =192) post caesarean section women was required to account for post randomisation exclusions or protocol violations.

Study subjects: Between October 2001 and February 2002. 192 women who underwent an emergency or elective caesarean section were enrolled in this trial study. Ninety six $(n=96)$ women were randomised to early feeding and ninety five $(n=96)$ to routine feeding group.

Eligibility criteria: Only HIV negative women who were scheduled to undergo caesarean section for various indications were enrolled in the study after informed consent. Women with severely obstructed labour with gross peritonitis and inevitable sepsis, severe uncontrolled medical conditions e.g. diabetes mellitus, severe pre-eclampsia/eclampsia where the patient needed intensive care in labour suite, very severely ill and or moribund patients and those not tested-for HIV infection.

Randomisation procedure: Assignment to one of the two study groups, "early feeding" or "routine feeding" was done randomly according to a computer - generated randomisation list (Random Number Generator, MicrosofteExcel 5.0). The group name was placed in sealed, consecutively numbered opaque envelopes. The sealed envelopes were secured and placed in the postoperative ward from where they were drawn consecutively until completion of the study. Neither the surgeon nor the 
investigators were aware of the study assignment. Patient information and consent forms were available separately.

Interventions: Recruitment and informed, consent. was gained prior to the operation. Randomisation to the "early feeding or routine feeding" groups was done after the caesarean section in the postnatal ward. An explanation to the study protocol was discussed with the patient to minimise protocol violations.

After randomisation, a $500 \mathrm{mls}$ packet of UHT milk was available to the trial group. The trial group (early feeding) was encouraged to take oral sips of water within 6 to 8 hours after the caesarean section. The first oral feeding of full milk (at least 150 $\mathrm{mls}$ at a time) or soup (if one did not take milk), was taken; by the mothers under supervision by the research assistants within 8 to 12 hours postoperatively, and thereafter, every 6 hours. A regular low-residue diet was introduced upon patients' 'demand after the first 24 hours

The "routine feeding" group were managed in the usual manner by restricting oral "intake 'for the 'first 24 hours and administration of sips of water 24 to 48 hours postoperatively. This decision was judged by the abdominal examination findings of the, absence of distension and the,presence of bowel sounds. A regular adult diet was allowed subsequently upon passage of flatus or stool (4).

Women remained on their intravenoüs infusion of fluids following the operation and had it discontinued as assessed by their haemodynamic stability. Once randomised, the observation charts were started and data on the gastrointestinal function were recorded prospectively. The participants were assessed at least twice a day, (morning and evening) by the research assistants who were house officers during the first 48 hours. They were required to elicit and document the outcome variables in unbiased and uniform fashion. Upon discharge, mothers were interviewed regarding their hospital progress and satisfaction with the study protocol.

Outcome measures: Multiple variables were studied in comparing the women in the two study groups. These included maternal demographic characteristics and outcome variables such as rate of ileus symptoms (nausea, vomiting, abdominal distention and diarrhoea) Other variables were postoperative time interval to presence of bowel sounds, passage of flatus and bowel movement, time interval to return to regular diet, maternal pyrexia and infection and acceptability and benefits of the early feeding.

Statistical analysis: Data were entered into Epi-info database. which was subsequently analysed using Epi-info and STATA computer software. Categorical data were analysed by Chi-square or fisher exact test and the Mann Whitney test was used. $P$-value $<0.05$ was determined to be statistically significant as appropriate.

Quality control: The standard questionnaire was pre-tested before executing the study. The house officers and nurses who took care of the patients participating in the study received training regarding the research protocols. The patient's caregiver(s) or attendant(s) also received briefing regarding the feeding regimens assigned The study was carried out in only one postnatal ward. This was intended to minimise breech of protocol and to ensure strict and closer follow-up by the investigator. The principal investigator was blinded and did not participate in the assessment of the outcome parameters to reduce bias.
Ethical considerition: Informed written consent was obtained from every patient before enrolment. They were informed of some possible complications that may arișe, In case any arise thatneeds attention, she will have promptand appropriate treatment.

\section{RESULTS}

Both groups did not differ significantly in mean maternal age, gravidity, or gestational age.

Table 1

General characteristics of the study subjects

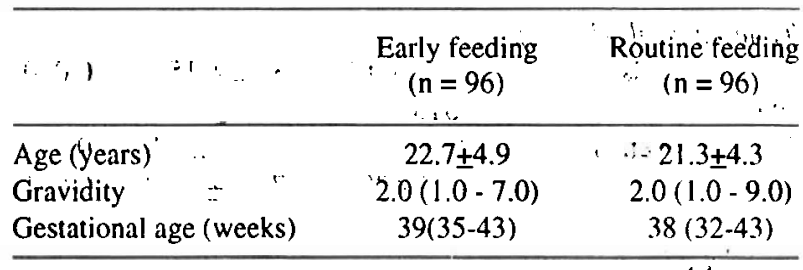

Table 2

Operative characteristics

\begin{tabular}{|c|c|c|c|}
\hline $1 !$ & $\begin{array}{c}\text { Early } \\
\text { feeding } \\
(n=96)\end{array}$ & $\begin{array}{c}\text { Routine } \\
\text { feeding } \\
(n=96)\end{array}$ & Significánce \\
\hline \multicolumn{4}{|l|}{ Type of anaesthesia } \\
\hline General & $95(99)$ & 94 (98.9.) & NS \\
\hline Regional & $(1.0)$ & (1.1) & NS \\
\hline \multicolumn{4}{|l|}{ Indication for $\mathrm{C} / \mathrm{S}$} \\
\hline Emergency & $92(95.8)$ & $91(95.8)$ & NS \\
\hline Elective & $4(4.2)$ & $4(4.2)$ & NS \\
\hline \multicolumn{4}{|l|}{ Type of $\mathrm{C} / \mathrm{S}$} \\
\hline Standard & $75(78.1)$ & $74(77.9)$ & $\therefore$ NS \\
\hline Misgav. L & $\therefore 21(21.9)$ & $21(22.1)$ & NS \\
\hline \multicolumn{4}{|l|}{ Uterine incision } \\
\hline Low transverse & $95(99.0)$ & $95(99.0)$ & NS \\
\hline Classical & $1(1.0)$ & $0(0.0)$ & NS \\
\hline \multicolumn{4}{|c|}{ Complications during surgery } \\
\hline Haemostasis & $1(1.0)$ & $I(1.1)$ & NS \\
\hline Adhesions & $4(4.2)$ & $2(2.1)$ & NS \\
\hline \multicolumn{2}{|c|}{ Duration of Surgery $(\mathrm{min}) 35.3 \pm 8.6$} & $35 \pm 8.6$ & NS \\
\hline
\end{tabular}

Women in both groups had similar type of anaesthesia, caesarean type and indications, complications during surgery and intra-operative findings, and mean duration of surgery.

Tables 3

Postoperative Characteristics

\begin{tabular}{lccc}
\hline & $\begin{array}{c}\text { Early } \\
\text { feeding } \\
(n=96)\end{array}$ & $\begin{array}{c}\text { Routine } \\
\text { feeding } \\
(n=96)\end{array}$ & p-value \\
\hline Postoperative complications: & $1(1.0)$ & $3(3.2)$ & NS \\
Fever $\left(>38^{\circ} \mathrm{C}\right)$ & $2(2.1)$ & $6(6.3)$ & NS \\
$\quad$ Wound sepsis & $5.0 \pm 1.1$ & $7.0 \pm 1.5$ & \\
No. of bottles of I.V fluids & & & \\
0.000 & $5.5 \pm 3.0$ & $6.0 \pm 3.8$ & NS \\
Length of hospital stay (days) & $15.1 \pm 7.0$ & $17.8 \pm 7.6$ & \\
Patient mobilisation (hours) & & & \\
0.001 & & & \\
\hline
\end{tabular}


The difference in the number of bottles of intravenous fluids received by patients in the two groups was statistically significant $(p=0.000)$. Mobilisation of women in the early feeding group was faster, $15.1 \pm 7.0$ versus $17.8 \pm 7.6$ hours $(p=0.001)$.

\section{Table 4}

Gastrointestinal parameters

\begin{tabular}{lccc}
\hline & $\begin{array}{c}\text { Early } \\
\text { feeding } \\
(\mathrm{n}=96)\end{array}$ & $\begin{array}{c}\text { Routine } \\
\text { feeding } \\
(\mathrm{n}=96)\end{array}$ & $\mathrm{p}$-value \\
\hline Time intervals (hours) & & & \\
Bowel sounds & $24.2 \pm 13$ & $34.2 \pm 15.6$ & 0.000 \\
Flatus & $51.6 \pm 18$ & $62.1 \pm 18.7$ & 0.000 \\
Bowel movement & $67.8 \pm 22.8$ & $75.8 \pm 22.4$ & 0.004 \\
Regular diets & $66.1 \pm 20.6$ & $75.1 \pm 18.3$ & 0.001 \\
& & & \\
Paralytic ileus symptoms & & & \\
None & $81(84.4 \%)$ & $67(70.5 \%)$ & \\
Nausea & $8(8.3 \%)$ & $14(14.7 \%)$ & 0.17 \\
Vomiting & $2(2.1 \%)$ & $3(3.2 \%)$ & 0.64 \\
Diarrhoea & $2(2.1 \%)$ & $4(4.2 \%)$ & 0.40 \\
Distention & $3(3.1 \%)$ & $7(7.4 \%)$ & 0.19 \\
\hline
\end{tabular}

Compared with those on routine feeding regimen, women in the early feeding group had a significantly more rapid $(\mathrm{p}=0.001)$ return to regular oral diets 66.1 versus 75.1 hours, and a significantly shorter $(p=0.004)$ mean interval to first bowel movement, 67.8 versus 75.8 hours. Time to development of bowel sounds and time to passage of flatus were significantly shorter ( $p$-values $=0.000$ ) in the early feeding group than those on routine feeding schedule.

The rate of gastrointestinal morbidity, including nausea, vomiting diarrhoea, or abdominal distension, was not significantly different in the two groups studied (15.6\% versus $29.5 \%$ ).

Acceptability of early initiation of oral feeding: The women were interviewed regarding their perception of the feeding protocol to which they were randomised and the pace at which they resumed their regular activities. In the early feeding group, all 96 (100\%) were highly satisfied with the availability of the UHT milk in the immediate postoperative period. Their acceptance of the UHT milk and the protocol was excellent. One mother in the early feeding, group did not enjoy the milk (instead took porridge) because she does not normally drink milk. Sixty three $(65.6 \%)$ of the mothers, said they would choose or recommend the early feeding protocol if they ever had another caesarean delivery. Fifty eight $(60.4 \%)$ of the mothers who were having repeat Caesarean section reported better experience with the early feeding protocol compared with the routine. Among women randomised to the routine feeding, 94 (99\%) expressed their desire for early introduction of oral feeding.
The effect of time of initiating oral feeding on lactation: When milk flow was compared between the two groups at the mean postoperative day $5 \pm 1.5$, there was no significant difference in the two groups (100\%, versus $97.9 \%$ ). However, more mothers in the early feeding group were significantly more satisfied $(p=0.000)$ with the amount of breast milk production and reported it was quite enough for the baby than their counterparts in the routine feeding group at the same period of time (82.3\% versus $37.9 \%)$.

\section{DISCUSSION}

Traditionally, obstetricians routinely withhold oral feeding during the postoperative period until resolution of the postoperative ileus, as demonstrated by passage of flatus. Recent studies in women undergoing Caesarean section have refuted this historical dictum and has demonstrated that early initiation of oral feeding seems to be well tolerated and even beneficial to patients $(2,8,9,14)$. Most of these studies were carried out in well-equipped specialised hospitals in developed countries and the majority of those Caesarean sections were performed under regional anaesthesia. In this present study $99 \%$ of the caesarean sections were performed under general anaesthesia and endotracheal intubation with only $1.0 \%$ under regional anaesthesia. This is because in Mulago hospital we traditionally do Caesarean sections under general anaesthesia.

This study demonstrated that there was no significant difference in the incidence of the usually feared paralytic ileus symptoms in the early feeding group ( $15.6 \%$ versus $29.5 \%$ ). It has also shown that women in the early feeding group had a more rapid return of their bowel function, with a significantly shorter mean postoperative time intervals to bowel sounds ( 24.2 hours versus 34.2 hours), passage of flatus ( 51 .6 hours versus 62.1 hours) and bowel movement (67.8 hours versus 75.8 hours). Our findings were in agreement with other studies on early and traditional feeding (9) in a prospective study of 221 patients to assess the gastrointestinal function and patient acceptability of early initiation of oral feeding after caesarean delivery found no significant increase in gastrointestinal morbidity $17.4 \%$ versus $15.6 \%$. Patolia et al. (2), in a randomised trial of 120 women delivered by Caesarean section under regional anaesthesia, observed no significant increase in incidence of ileus symptoms in both groups $31.7 \%$ versus $26.7 \%$.

This study demonstrated that early postoperative feeding did not appear to increase the incidence of postoperative paralytic ileus or of gastrointestinal symptoms as usually feared. In fact, women who were fed earlier were more likely to report an absence of any symptoms associated with ileus, although this difference was not statistically significant. It is evident from our study and other previous studies $(1,15)$, that the positive effect on the gastrointestinal tract from such stimulation by early oral feeding decreased the length of postoperative ileus. In this study it was evident that women who were 
offered food early were generally satisfied with eating earlier. This positive observation demonstrated by this study regarding tolerance and acceptability of early postoperative feeding are well supported by physiological knowledge (1). Our study has also shown that women in the early feeding group had a significantly faster resumption of regular normal diets than the routine group ( 66.1 hours versus 75.1 hours).

This study was able to demonstrate that early feeding was well tolerated and accepted. Similar results have been reported in earlier studies $(9,13,14)$ though return to regular diets in those studies were sooner and more rapid than in the present study. This may be attributable to some patients not eating even if they did well. Furthermore, the patient's attendants and often their fellow patients actively discouraged them to eat as soon as possible. It became evident at the initial stages of this study that patients, their attendants and often some hospital staff believed that eating earlier would lead to complications and there was initial difficulty in complying with the interpretation of the research protocol. Later this perception was successfully corrected.

This study also demonstrated that women who received food earlier made a more rapid recovery and expressed their interest in earlier hospital discharge. The findings significantly indicated that women in the early feeding group got out of bed (patient mobilisation) earlier $(p=0.001)$ than their control group ( 15.1 hours versus 17.8 hours). This could probably have been because of the adequate rehydration and improved early energy intake and the shorter duration of intravenous line, which would usually keep postoperative patients on their beds. When compared with women who were on routine feeding, those who were fed early required less number of bottles of intravenous fluids ( 5.0 bottles versus 7.0 bottles). The average hospital stay was similar and not statistically significant in both groups ( 5.5 days versus 6.0 days). In all cases, the extended hospitalisation was for reasons other than postoperative ileus. The duration of hospitalisation was determined by the departmental protocol. However women who were on early feeding made a faster recovery and expressed their interest in earlier discharge. The economic advantage of this regimen versus the routine is apparent.

The incidence of major postoperative infections, including fever $\left(<38^{\circ} \mathrm{C}\right)$ and wound sepsis in the two study groups were comparable and not statistically significant. The majority of postoperative infections were wound sepsis. One woman, who was on the routine feeding regimen developed severe wound sepsis with dehiscence, her condition resolved with daily dressing, antibiotics and secondary suture.

This study did elucidate the effect of time of initiating oral feeding on the establishment of succesful lactation. However, based on the results of this study, it was apparent that there was no considerable variability, in the initiation of lactation in the immediate postoperative period. However, although we did not attempt to evaluate the quantity of milk production, there was a significant number of mothers in the early, feeding group who were satisfied with the amount of breast milk compared with those in the routine group ( $82.3 \%$ versus $37.9 \%$ ) when assessed at a mean day of $5.0 \pm 1.5$. Based on this results, it is reasonable to say, that early initiation of oral feeding has the potential for improving successful lactation.

This study had some limitations. Firstly, because of the nature of the intervention, neither the women nor the monitors were blinded to the feeding assignment: this fact may introduce a bias and thus affect the assessment of subjective complaints such as nausea and abdominal cramping. The fact that both the study group and the controls lived on the same ward could have affected compliance. This in addition could have been confounded by the providers who were not part of the study team who could have influenced patients especially in the early part of the study. Lastly, the return of the bowel function as demonstrated by passage of flatus and a bowel movement was based on the patient's recall of hours of the happenings likewise presence of the bowel sounds was based on a single time point at which the abdomen was auscultated. Abdomens were auscultated twice a day. which meant that the durations were overestimated.

In conclusion, early initiation of oral feeding after caesarean delivery is safe and well tolerated and can be implemented without an increase in gastrointestinal symptoms or paralytic ileus. Early initiation of oral feeding after caesarean delivery is associated with a more rapid return of normal bowel function, faster patient mobilisation and faster resumption of normal regular diets. This approach may be associated with a shorter hospital stay, and may facilitate early hospital discharge. We recommend early initiation of oral feeding after Caesarean delivery should be recommended and offered to all women who have had uncomplicated labour and operations.

\section{REFERENCES}

1 Casto. J.C. Kramer, J. and Drake. J. Postoperative Feeding: A clinical Review. Obstet. Gynecol. Survey. 2000: 555: 571-573.

2. Patolia, D.S., Hilliard, R.I., Toy, E.C. et al. Early feeding after Caesarean: Randomised trial. Obstet. Gynecol. 2001: 98 : 113-116.

3. Clarke-Pearson, D.L. Olt, G.J. Rodriquez, G.C. et al. Preoperative evaluation and postoperative management. In Berek J. Adashi E. Hillard P.eds. Novak's Gynecology: 12th Ed. Baltimore: Williams and Wilkins. 1996; 571-572.

4. Horowitz, I.R. and Rock, J.A., Postanaesthesia and postoperative care. In: Thompson J.D. Rock J.A. eds. TeLinde's operative gynecology 8th edition. Philadelphia: J.B. Lippincott. 1997: 142163.

5. Nezhat, C., Nezhat, F.. and Nezhat, C. Operative Laparoscopy (Minimal invasive surgery) State of the art. J. Gynecol. Surg. 1992; 8:111-141.

6. Tadir Y., and Fisch B. Operative laparoscopy: A challenge for general gynaecology? Am. J. Obstet. Gynecol. 1993: 169:7-12.

7. Binderow. S.R. Cohen. S.M. Wexner. S.D. el al. Must early post operative oral intake be limited to laparoscopy? Dis. Colon Rectum. 1994: 37:584-589. 
8. Burrows, W.R., Gingo, Jr. A.J., Rose S.M. et al. Safety and efficacy of early postoperative solid food consumption after Caesarean section. J. Reprod. Med. 1995; 40:463-467.

9. Soriano, D. Dulitzki, M. Keider, N. et al. Early oral feeding after Caesarean delivery. Obstet. Gynecol. 1996; 87:10061008.

10. Clevers, G.J., and Smout A.J. The natural course of postoperative ileus following abdominal surgery. Neth. $J$. Surg. 1989; 41:97-99.

11. Bowling, T.E. Does disorder of gastrointestinal motility affect food intake in the post-surgical patient? Proc. Nutr. Soc. 1994; 53: 15!-157.
12. Strong, T.H., Jr. Brown, W.L., Jr. and Brown, W.L. et al. Experience with early post Caesarean hospital dismissal Am.J. Obstet. Gynecol.1993:169:116-119.

13. Weinstein, L., Dyne, P.L., and Duerbeck. N.B. The PROEF diet- A new postoperative regimen for oral early feeding. $\mathrm{Am}$. J. Obstet Gynecol. 1993: 168: 128-131.

14. Bufo, A.J., Feldman, S. Daniels, G.A et. al. Early postoperative feeding. Dis Colon Reclum. 1994; 37:1260-1265.

15. Kramer, R., Van Someren, J., Qualls, C. et al. Post operative management of Caesarean patients. The effect of immediate feeding on the incidence of ileus. Obstet. Gynecol. 1996; 88: 29-32.

\section{Do you need up-to-date peer reviewed medical literature?}

Kenya Medical Association, in collaboration with GlaxoSmithKline, is pleased to introduce

\section{KMA/GSK RESOURCE CENTRE}

A new state-of-the-art facility for frontline health workers, researchers and policy makers in Kenya to acess:

\section{CURRENT CRITICAL MEDICAL AND PUBLIC HEALTH INFORMATION DATABASES}

Relevant and reliable abstracts from leading medical journals available at the following affordable rates:

Literature Search (CD-ROM)

Printing
-Ksh. 50 per search

-Ksh. 10 per page

\section{ACCESS TO INFORMATION YOU CAN USE}

Address: KENYA MEDICAL ASSOCIATION

Chyulu Road, Upper Hill

P.O Box 41632, Code 00100, GPO, NAIROBI

E-mail: eamj@wananchi.com

Phone: 2712010

Fax: 